\title{
Effect of stacking-fault energy on the accumulation of dislocations during plastic deformation of copper-based polycrystalline alloys
}

\author{
N. A. Koneva ${ }^{\dagger}$, L. I. Trishkina, T. V. Cherkasova \\ †koneva@tsuab.ru \\ Tomsk State University of Architecture and Building, 2 Solyanaya Sq., 634003, Tomsk, Russia
}

\begin{abstract}
The transmission electron microscopy was used to investigate the dislocation structure and accumulation of dislocations during the plastic deformation in $\mathrm{Cu}-\mathrm{Al}$ and $\mathrm{Cu}-\mathrm{Mn}$ polycrystalline $\mathrm{FCC}$ solid solutions. $\mathrm{Al}$ content in $\mathrm{Cu}-\mathrm{Al}$ alloys varied from 0.5 to 14 at.\%, and $\mathrm{Mn}$ content in $\mathrm{Cu}-\mathrm{Mn}$ alloys varied from 0.4 to 25 at.\%. The alloy samples with the grain size ranging from 20 to $240 \mu \mathrm{m}$ were studied., They were subjected to tensile deformation at the strain rate of $2 \cdot 10^{-2} \mathrm{~s}^{-1}$ at temperatures $293-673 \mathrm{~K}$. Observations of the structure on thin foils were carried out on electron microscopes at $125 \mathrm{kV}$ accelerating voltage. For different strains, the scalar dislocation density and other parameters of the defect structure such as the size of the dislocation cells, density of microtwins etc. were measured. The results show that the increased content of $\mathrm{Mn}$ and $\mathrm{Al}$ in alloys is accompanied by an increase in the dislocation density. In $\mathrm{Cu}-\mathrm{Al}$ alloys the dislocation density depends on the stacking fault energy. With its decrease, the density of dislocations increases. An explanation of this behavior is given. In $\mathrm{Cu}-\mathrm{Mn}$ alloys the increased $\mathrm{Mn}$ content does not modify the stacking fault energy, while in $\mathrm{Cu}$ - $\mathrm{Al}$ alloys the dislocation density increases with the increase in the deformation temperature due to the temperature effect on the stacking fault energy. In $\mathrm{Cu}-\mathrm{Mn}$ alloys, the temperature reduces the dislocation density. The resistance to deformation both in $\mathrm{Cu}-\mathrm{Al}$ and $\mathrm{Cu}-\mathrm{Mn}$ alloys decreases with the temperature increase. Physical causes of the absence of the temperature anomaly of mechanical properties in $\mathrm{Cu}-\mathrm{Al}$ alloys are discussed.
\end{abstract}

Keywords: alloys, deformation, dislocation density, stacking-fault energy.

\section{Влияние энергии дефекта упаковки на накопление дислокаций при пластической деформации} поликристаллических сплавов на основе меди

\author{
Конева Н.А. ${ }^{\dagger}$, Тришкина Л.И., Черкасова Т.В.
}

Томский государственный архитектурно-строительный университет, пл. Соляная 2, 634003, Томск, Россия

\begin{abstract}
Методом просвечивающей дифракционной электронной микроскопии (ПЭМ) проведено исследование дислокационной структуры и накопления дислокаций при деформации поликристаллических ГЦК твердых растворов систем $\mathrm{Cu}-\mathrm{Al}$ и $\mathrm{Cu}-\mathrm{Mn}$. Содержание $\mathrm{Al}$ в сплавах $\mathrm{Cu}-\mathrm{Al}$ варьировалось от 0,5 до 14 ат.\%. Содержание $\mathrm{Mn}$ в сплавах $\mathrm{Cu}-\mathrm{Mn}$ изменялось в пределах 0,4...25 ат.\%. Изучены сплавы с размером зерен в интервале 20 - 240 мкм. Образцы сплавов деформировались растяжением со скоростью $2 \cdot 10^{-2} \mathrm{c}^{-1}$ до разрушения при температурах 293 - 673 К. Структура деформированных до различных степеней деформации образцов изучали на фольгах на электронных микроскопах при ускоряющем напряжении 125 кВ. Для разных степеней деформации измерялась скалярная плотность дислокаций и некоторые другие параметры дефектной структуры (размер дислокационных ячеек, плотность микродвойников и др.). В результате исследований установлено, что увеличение содержания $\mathrm{Mn}$ и $\mathrm{Al}$ в сплавах сопровождается увеличением плотности дислокаций. При этом в сплавах $\mathrm{Cu}-\mathrm{Al}$ плотность дислокаций зависит от энергии дефекта

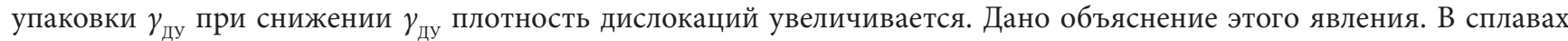
$\mathrm{Cu}-\mathrm{Mn}$ увеличение содержания $\mathrm{Mn}$ не изменяет $\gamma_{\text {ду }}$, соответственно этот эффект отсутствует. $\mathrm{B}$ сплавах Cu-Al обнаружено увеличение плотности дислокаций с повышением температуры деформации, что связано с влиянием температуры на величину $\gamma_{\text {ду. }}$ В сплавах $\mathrm{Cu}-\mathrm{Mn}$ увеличение температуры деформации снижает плотность дислокаций. Сопротивление деформированию как в сплавах $\mathrm{Cu}-\mathrm{Al}$, так и в сплавах с $\mathrm{Mn}$ с повышением температуры снижается. Обсуждаются физические причины отсутствия температурной аномалии механических свойств в сплавах Cu-Al.
\end{abstract}

Ключевые слова: сплавы, деформация, плотность дислокаций, энергия дефекта упаковки. 


\section{1. Введение}

В процессе пластической деформации металлических материалов происходит накопление дислокаций. Скалярная плотность дислокаций ( $\rho$ ) в поликристаллах ГЦК металлов и сплавов зависит от ряда факторов. Наиболее важными из них являются: 1) степень пластической деформации, 2) скорость деформации, 3) величина твердорастворного упрочнения, 4) температура деформации, 5) размер зерна поликристалла. Имеет место еще один фактор, который также может оказать влияние на накопление дислокаций. Этот фактор - величина энергии дефекта упаковки $\left(\gamma_{\text {ду }}\right)$. Влияние $\gamma_{\text {ду }}$ на величину $\rho$ изучено очень мало. Отметим в связи с этим некоторые работы.

В [1] было исследовано изменение плотности дислокаций в зависимости от $\gamma_{\text {ду }}$ при ударных нагружениях меди и сплавов $\mathrm{Cu}-\mathrm{Al} \mathrm{c}$ размером зерна 50 мкм. Для сравнения были проведены также испытания на сжатие со скоростью $5 \cdot 10^{-4} \mathrm{c}^{-1}$ до степени деформации $\varepsilon_{\text {ист }}=0.25$. Плотность дислокаций вычислялась из измерений запасенной энергии. В испытаниях кроме меди было четыре сплава с содержанием $\mathrm{Al}$ 0.2, 2, 4 и 6 вес.\%. Из полученных в [1] результатов следует, что влияние $\gamma_{\text {ду }}$ на накопление дислокаций зависит от величины нагрузки при ударном нагружении. При статическом нагружении

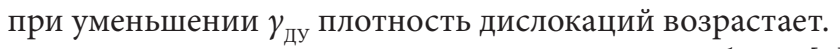

Интересные результаты приведены в работе [2] для $\mathrm{Al}, \mathrm{Ni}, \mathrm{Cu}$ и стали состава Fe-31Mn-3Al-3Si (вес. \%). Из анализа приведенных немногочисленных данных для чистых металлов и собственных результатов на стали авторы [2] делают заключение, что с уменьшением $\gamma_{\text {ду }}$ плотность дислокаций возрастает.

Величина $\gamma_{\text {ду }}$ оказывает влияние на накопление дислокаций ультрамелкозернистых (УМЗ) материалов. В [3] исследовалась $\mathrm{Cu}$ и сплавы $\mathrm{Cu}-\mathrm{Zn}$ с содержанием $\mathrm{Zn}$ 10 и 30 вес\%. Эти материалы имели $\gamma_{\text {ду }}$ соответственно 41, 22 и 7 мДж/ $\mathrm{m}^{2}$. УМЗ состояние достигалось кручением под гидростатическим давлением. Образцы затем подвергались растяжению при комнатной температуре. Рентгеновским методом была измерена плотность дислокаций. Было установлено, что с уменьшением $\gamma_{\text {ду }}$ плотность дислокаций увеличивается.

Скалярная плотность дислокаций является важным параметром дефектной структуры деформированного материала. Ее значение определяет дислокационный вклад в сопротивление движению дислокаций в металлах и сплавах. Поэтому важно знать, какие факторы и каким образом влияют на накопление дислокаций. Среди них - энергия дефекта упаковки (ЭДУ). Удобными объектами для решения этой задачи являются сплавы систем $\mathrm{Cu}-\mathrm{Al}$ и $\mathrm{Cu}-\mathrm{Mn}$. Увеличение содержания $\mathrm{Al}$ в сплавах $\mathrm{Cu}-\mathrm{Al}$ снижает $\gamma_{\text {ду }}$ [4], увеличение содержания $\mathrm{Mn}$ почти не изменяет $\gamma_{\text {ду }}$ [5]. Поэтому изучение дислокационной структуры деформированных сплавов систем $\mathrm{Cu}-\mathrm{Al}$ и $\mathrm{Cu}-\mathrm{Mn}$ может выделить влияние $\gamma_{\text {ду }}$ на накопление дислокаций при деформации.

Целью настоящей работы было исследование влияния энергии дефекта упаковки на накопление дислокаций при деформации поликристаллических сплавов на основе меди.

\section{2. Материал и методы исследования}

Исследовались поликристаллические ГЦК твердые растворы системы $\mathrm{Cu}-\mathrm{Al}$ с содержанием $\mathrm{Al}$ от 0.5 до 14 ат. \% и системы $\mathrm{Cu}-\mathrm{Mn}$, в которых концентрация $\mathrm{Mn}$ изменялась в пределах $0.4 \ldots 25$ ат. \%. Средний размер зерен в сплавах был 20...240 мкм. Образцы сплавов подвергались растяжению со скоростью $2 \cdot 10^{-2} \mathrm{c}^{-1}$ до разрыва при температурах $293 \mathrm{~K}-673 \mathrm{~K}$ на машине «Instron». Структуру деформированных до различных степеней деформации образцов изучали на фольгах на электронных микроскопах ЭМB-100AK и Tesla BS-540, снабженных гониометром с наклоном и прецессией. Сначала изучалась качественная картина дефектной структуры, затем хорошо известным методом секущей [6] измерялась скалярная плотность дислокаций и другие параметры субструктуры (размер дислокационных ячеек, плотность микродвойников и др.). Результаты измерений подвергались статистической обработке.

\section{3. Результаты исследования и их обсуждение}

На рис. 1 приведены значения средней скалярной плотности дислокаций $\rho$ в сплавах на основе меди со средним размером зерен $d=60$ мкм, деформированных при комнатной температуре $T=293 \mathrm{~K}$ в вависимости от концентрации $\mathrm{Mn}$ (рис. 1a) и $\mathrm{Al}$ (рис. 1b). Из рисунков следует, что с увеличением концентрации как $\mathrm{Mn}$, так и $\mathrm{Al}$ плотность дислокаций увеличивается при всех степенях деформации. При этом увеличение содержания $\mathrm{Al}$ приводит к более интенсивному накоплению дислокаций.
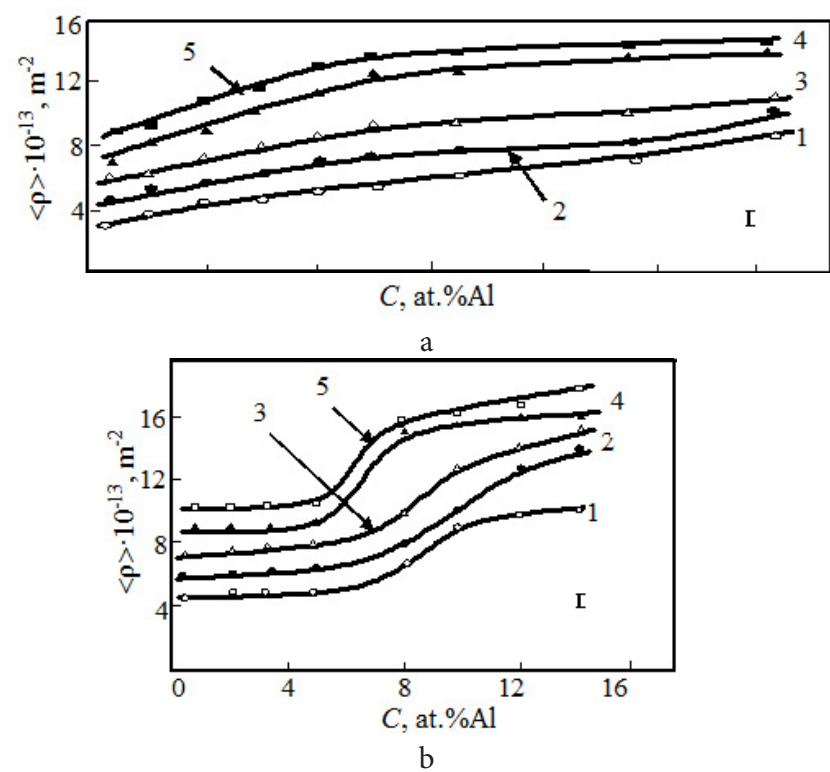

Рис. 1. Зависимости средней скалярной плотности дислокаций $\rho$ от концентрации $C$ легирующего элемента $\mathrm{Mn}$ (a) и $\mathrm{Al}(\mathrm{b})$ при разных степенях деформации $\varepsilon_{\text {ист }}: 1-0.05$, $2-0.10,3-0.20,4-0.30,5-0.40$. Размер зерна $d=60$ мкм.

Fig. 1. Dependences of the mean scalar dislocation density $\rho$ on the concentration $C$ of alloying elements $\mathrm{Mn}$ (a) and $\mathrm{Al}$ (b) at different strains $\varepsilon_{\text {true }}: 1-0.05,2-0.10,3-0.20,4-0.30,5-0.40$. Grain size $d=60 \mu \mathrm{m}$. 
Увеличение содержания как $\mathrm{Mn}$, так и $\mathrm{Al}$ в сплавах создает твердорастворное упрочнение. В ГЦК твердых растворах предел текучести в основном определяется величиной твердорастворного упрочнения $\tau_{f}$ [7]. Вклад ближнего порядка оказывается незначительным. Величина $\tau_{f}$ в процессе деформации остается неизменной. В связи с вышесказанным, величина твердорастворного упрочнения $\tau_{f}$ для исследуемых сплавов была определена с использованием значений предела текучести $\sigma_{0.2}[8]$, а именно, $\tau_{f}=\sigma_{0.2} / m$, где $m=3.1-$ ориентационный множитель. На рис. 2 приведены зависимости $\tau_{f}$ для исследуемых сплавов в испытаниях при комнатной температуpe $T=293$ К. Видно, что для всего исследуемого спектра размеров зерен поликристаллов $\tau_{f}$ для сплавов с $\mathrm{Mn}$ несколько выше, чем для сплавов с Al. Другими словами, увеличение содержания $\mathrm{Al}$ в сплавах и, следовательно, увеличение твердорастворного упрочнения не может объяснить более интенсивное накопление дислокаций в них по сравнению со сплавами $\mathrm{Cu}-\mathrm{Mn}$.

Увеличение содержания $\mathrm{Al}$ в сплавах меди уменьшает $\gamma_{\text {ду }}[4]$, увеличение содержания $\mathrm{Mn}$ практически не изменяет $\gamma_{\text {ду }}$ [5]. В связи с этим было изучено влия-

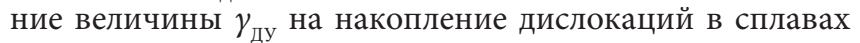
$\mathrm{Cu}-\mathrm{Al}$. На рис. 3 представлены зависимости $\rho$ от $\gamma_{\text {ду }}$ для сплавов $\mathrm{Cu}-\mathrm{Al}$ с разным размером зерен, испытанных при $T=293$ К. Видно, что во всех случаях уменьше-

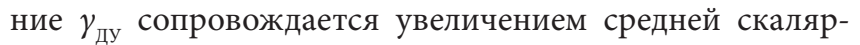
ной плотности дислокаций. Это особенно проявляется при $\gamma_{\text {ду }}<30$ мДж $\cdot \mathrm{M}^{-2}$. Можно полагать, что увеличение

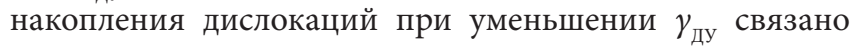
с уменьшением вероятности выхода дислокаций из плоскости скольжения. К аналогичному заключению пришли авторы [2].

Накопление дислокаций изучалось также в сплавах, деформированных при температурах (T) выше комнатной вплоть до $673 \mathrm{~K}$. На рис. 4 для сплава $\mathrm{Cu}+6$ ат. $\% \mathrm{Mn}$ представлены зависимости $\rho=f(T)$ для различных степеней деформации и разных размеров зерен. Средняя плотность дислокаций уменьшается с увеличением температуры. Подобные зависимости наблюдаются и для других сплавов системы $\mathrm{Cu}-\mathrm{Mn}$. Такое поведение плотности дислокаций можно было ожидать, исходя из общих представлений о влиянии температуры на релаксацию внутренних напряжений деформированных материалов [9].

Другое поведение наблюдалось для сплавов системы Cu-Al. На рис. 5 представлены зависимости $\rho=f(T)$ для различных степеней деформации $\varepsilon_{\text {ист }}$ сплавов $\mathrm{Cu}+10$ ат. \% $\mathrm{Al}$ и $\mathrm{Cu}+14$ ат.\% Al. Как видно из рис. 5, $\rho$ растет с увеличением температуры деформации. Аналогичные зависимости были получены для других сплавов системы $\mathrm{Cu}-\mathrm{Al}$. Изменение размера зерен качественно не изменяет характер зависимостей $\rho=f(T)$. Исходя из представленных выше результатов (см. рис. 3), свидетельствующих о том, что уменьшение $\gamma_{\text {ду }}$ способствует накоплению дислокаций при деформации сплавов системы $\mathrm{Cu}-\mathrm{Al}$, можно было полагать, что повышение

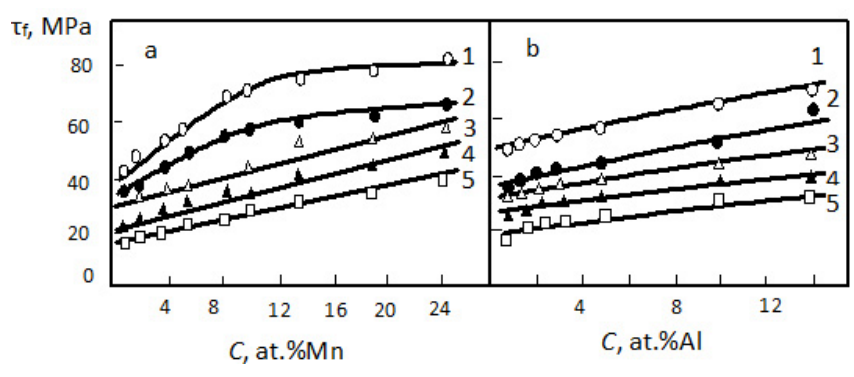

Рис. 2. Зависимости величины твердорастворного упрочнения $\tau_{f}$ от концентрации $C$ легирующего элемента $\mathrm{Mn}(\mathrm{a})$ и $\mathrm{Al}$ (b) при разных размерах зерен $d$, мкм: $1-20,2-40,3-60$, $4-120,5-240 . T=293 \mathrm{~K}$.

Fig. 2. Dependences of solid solution strengthening $\tau_{f}$ on the concentration $C$ of alloying elements $\mathrm{Mn}$ (a) and $\mathrm{Al}(\mathrm{b})$ at different grain sizes, $\mu \mathrm{m}: 1-20,2-40,3-60,4-120,5-240 . T=293 \mathrm{~K}$.

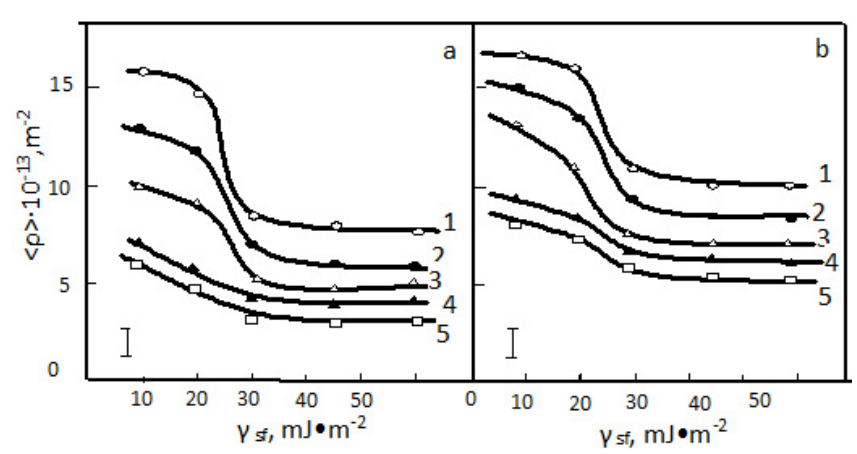

Рис. 3. Зависимости средней скалярной плотности дислока-

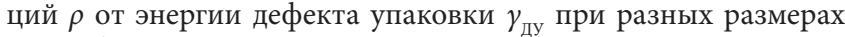
зерен $d$, мкм: $1-20,2-40,3-60,4-120,5-240$. Степень деформации: $\mathrm{a}-\varepsilon_{\text {ист }}=0.05 ; \mathrm{b}-\varepsilon_{\text {ист }}=0.10 . T=293$ К. Сплавы системы Cu-Al.

Fig. 3. Dependences of the mean scalar dislocation density $\rho$ on the stacking-fault energy $\gamma_{\text {sf }}$ in $\mathrm{Cu}-\mathrm{Al}$ alloys at different grain sizes $d$, $\mu \mathrm{m}: 1-20,2-40,3-60,4-120,5-240$. $\mathrm{a}-\varepsilon_{\text {true }}=0.05 ; \mathrm{b}-\varepsilon_{\text {true }}=0.10 . T=293 \mathrm{~K}$.

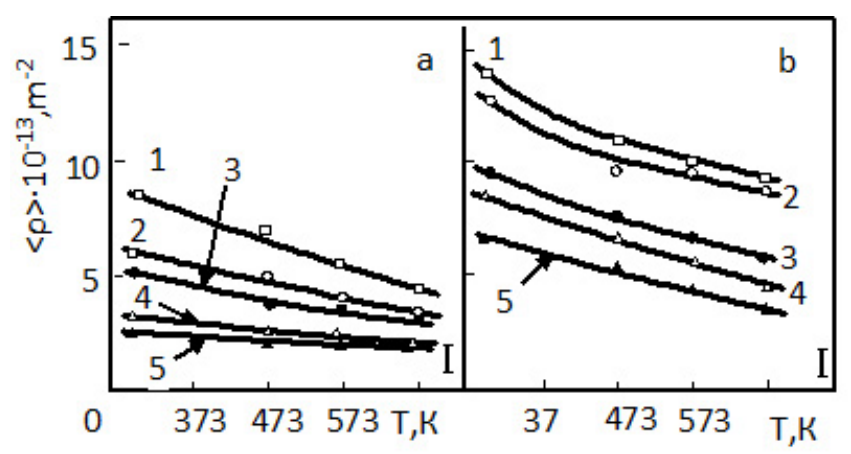

Рис. 4. Зависимости средней скалярной плотности дислокаций $\rho$ от температуры испытания $T$ в сплаве $\mathrm{Cu}+6$ ат. $\% \mathrm{Mn}$ при разных степенях деформации: $\left(\mathrm{a}-\varepsilon_{\text {ист }}=0.05 ; \mathrm{b}-\varepsilon_{\text {ист }}=0.30\right)$ и разных размерах зерен $d$, мкм: $1-20 ; 2^{\text {ист }}-40 ; 3-60 ; 4-120$; $5-240$.

Fig. 4. Dependences of the mean scalar dislocation density $\rho$ on temperature $\mathrm{T}$ in $\mathrm{Cu}+6$ at. $\% \mathrm{Mn}$ alloy at different deformation degrees $\varepsilon_{\text {true }}: \mathrm{a}-0.05 ; \mathrm{b}-0.30$, and different grain size $d, \mu \mathrm{m}$ : $1-20 ; 2-40 ; 3-60 ; 4-120 ; 5-240$. 


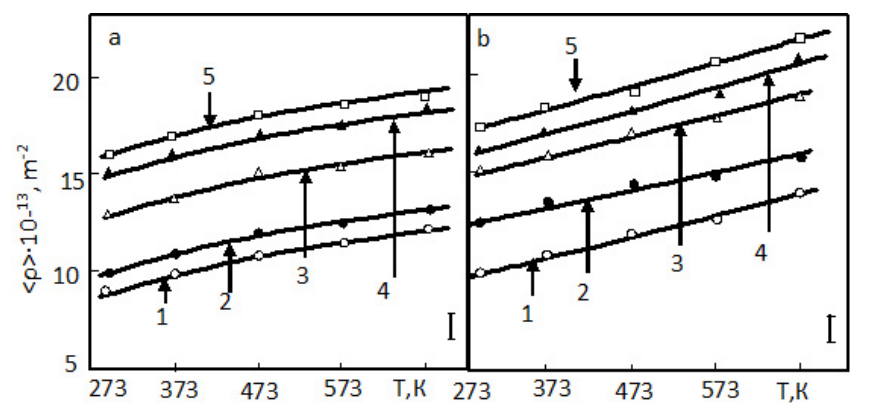

Рис. 5. Зависимости средней скалярной плотности дислокаций $\rho$ от температуры испытания $T$ в медно-алюминиевых сплавах $\mathrm{Cu}+10$ ат. \% $\mathrm{Al}$ (a) и $\mathrm{Cu}+14$ aт. \% $\mathrm{Al}$ (b) при разных степенях деформации $\left(\varepsilon_{\text {ист }}\right): 1-0.05 ; 2-0.10 ; 3-0.20 ; 4-0.30$; $5-0.40$. Размер зерна $d=60$ мкм.

Fig. 5. Dependences of the mean scalar dislocation density $\rho$ on the test temperature $\mathrm{T}$ in $\mathrm{Cu}+10$ at. $\% \mathrm{Al}(\mathrm{a})$ and $\mathrm{Cu}+14$ at. $\% \mathrm{Al}(\mathrm{b})$ alloys at different deformation degrees $\varepsilon_{\text {true }}: 1-0.05 ; 2-0.10$; $3-0.20 ; 4-0.30 ; 5-0.40$. Grain size $d=60 \mu \mathrm{m}$.
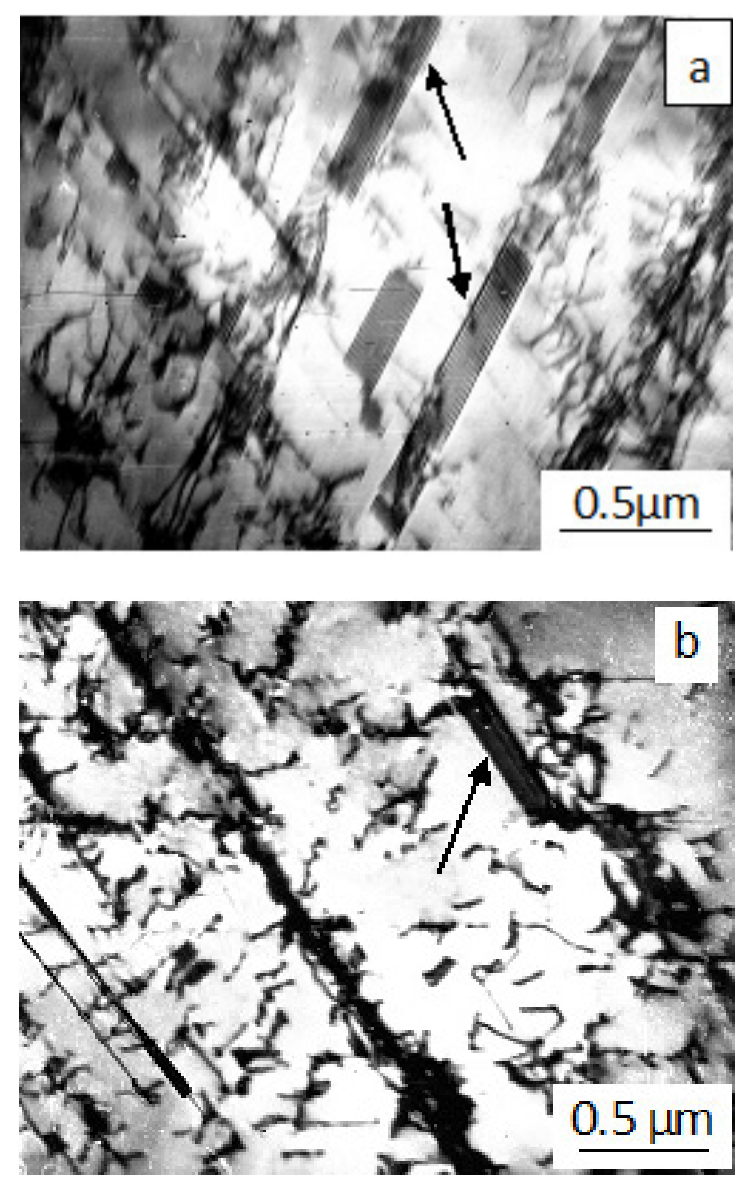

Pис. 6. Дефекты упаковки в сплаве $\mathrm{Cu}+14 \mathrm{at} \% \mathrm{Al}$, деформированном при разных температурах испытания: $\mathrm{a}-T=373 \mathrm{~K}, \varepsilon_{\text {ист }}=0.05 ; \mathrm{b}-T=573 \mathrm{~K}, \varepsilon_{\text {ист }}=0.15$. Плоскость фольги (110). Стрелками указаны дефекты упаковки.

Fig. 6. Stacking-fault defects in $\mathrm{Cu}+14$ at. $\% \mathrm{Al}$ alloy subjected to deformation at different temperatures: a $-T=373 \mathrm{~K}, \varepsilon_{\text {true }}=0.05$; $\mathrm{b}-T=573 \mathrm{~K}, \varepsilon_{\text {true }}=0.15$. Foil plane is (110). Arrows indicate stacking faults.

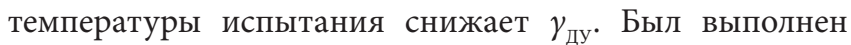
анализ дислокационных структур, наблюдаемых в сплавах при разных температурах деформации. Установлено, что повышение температуры деформации приводит к возникновению протяженных дефектов упаковки (ДУ). Примеры наблюдений ДУ в сплавах $\mathrm{Cu}+10$ ат. \% $\mathrm{Al}$ и $\mathrm{Cu}+14$ ат. \% $\mathrm{Al}$ при повышенных температурах испытания приведены на рис. 6. Полученные результаты

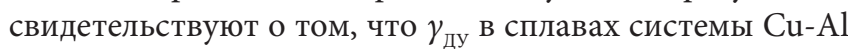
уменьшается при повышении температуры деформа-

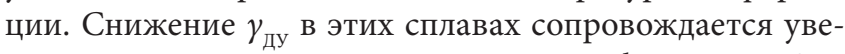
личением плотности дислокаций при деформации. Это объясняет тот факт, что плотность дислокаций в сплавах системы $\mathrm{Cu}-\mathrm{Al}$ увеличивается с ростом температуры деформации.

В связи с полученными результатами представляется важным обсудить следующее. Повышение температуры деформации снижает напряжение течения как в сплавах $\mathrm{Cu}-\mathrm{Mn}$, так и в сплавах $\mathrm{Cu}-\mathrm{Al}$. Возникает естественный вопрос, почему повышение плотности дислокаций при увеличении температуры деформации не вызывает аномального эффекта влияния температуры на сопротивление деформированию в сплавах системы $\mathrm{Cu}-\mathrm{Al}$. Объяснение этого явления заключается в том, что сопротивление деформированию имеет комплексный характер [7]. Скалярная плотность дислокаций $\rho$ создает вклад равный

$$
\Delta \sigma_{g}=m \alpha G b \sqrt{\rho},
$$

где $\alpha-$ параметр междислокационного взаимодействия, $G$ - модуль сдвига, $b$ - вектор Бюргерса. Между тем в исследуемых твердых растворах имеются вклады в сопротивление деформированию, связанные с формированием различных субструктур и создающих субструктурное упрочнение. Так, при небольших концентрациях $\mathrm{Al}$ (менее 8 ат.\%) в сплавах $\mathrm{Cu}-\mathrm{Al}$ на значительном интервале степеней деформации создается и развивается ячеистая субструктура [10]. Размер дислокационных ячеек с повышением температуры увеличивается, что, в соответствии с соотношением Стэкера - Холта [11] уменьшает вклад ячеистой субструктуры в сопротивление деформированию.

В сплавах меди с высокой концентрацией $\mathrm{Al}$ (более 8 ат.\%) в процессе деформации происходит микродвойникование. Включение микродвойникования как дополнительной моды пластической деформации уменьшает скорость деформационного упрочнения [12]. Еще один вклад в сопротивление деформированию - вклад внутренних напряжений. Их оценка показала, что вклад внутренних напряжений с увеличением температуры деформации снижается.

Таким образом, хотя средняя скалярная плотность дислокаций с повышением температуры испытания в сплавах $\mathrm{Cu}-\mathrm{Al}$ увеличивается, комплексный характер их упрочнения объясняет уменьшение сопротивления деформированию с повышением температуры, то есть отсутствие температурной аномалии механических свойств. 


\section{4. Заключение}

В работе методом ПЭМ проведено исследование дислокационной структуры и накопления дислокаций при деформации поликристаллических ГЦК твердых растворов систем $\mathrm{Cu}-\mathrm{Al}$ и $\mathrm{Cu}-\mathrm{Mn}$. В результате установлено следующее:

1. Величина скалярной плотности дислокаций в деформированных сплавах $\mathrm{Cu}-\mathrm{Al}$ зависит от $\gamma_{\text {ду. }}$ С уменьшением $\gamma_{\text {ду }}$ скалярная плотность дислокаций возрастает. Увеличение $\rho$ связано с уменьшением вероятности выхода дислокаций из плоскости скольжения. Повышение температуры деформации сопровождается увеличением $\rho$ в сплавах $\mathrm{Cu}-\mathrm{Al}$, что связано с уменьшением $\gamma_{\text {ду. }}$. В сплавах $\mathrm{Cu}-\mathrm{Mn} \rho$ уменьшается с ростом температуры.

2. Сопротивление деформированию в сплавах $\mathrm{Cu}-\mathrm{Al}$ с ростом температуры испытания снижается, хотя $\rho$ растет. Такое поведение обусловлено комплексным характером упрочнения исследуемых твердых растворов.

Благодарность/Acknowledgements. Исследования выполнены при поддержке государственного задания на проведение научных исследований по проекту № 3.8320.2017/БЧ.

\section{Литература/References}

1. A. Rohatgi, K. S. Vecchio. Mat. Sci. Eng. A. 328, 256-266 (2002)

2. G. Dini, R. Ueji, A. Najafizadeh, S. M. Minir - Vaghefi. Mat. Sci. Eng. A. 527, 2759-2763 (2010)

3. Y.H. Zhao, X. Z. Liao, Z. Horita, T. G. Langdon, Y. T. Zhu.
Mat. Sci. Eng. A. 493, $123-129$ (2008)

4. S. Crampin, D. D. Vedensky, R. Monnier. Phil. Mag. A. 67 (6), 1447 - 1457 (1993)

5. Th. Steffens, Ch. Schwink, A. Korner, H.P. Karnthaler. Phil. Mag. A. 56 (2), $161-173$ (1987)

6. K.S. Chernyavskii, Stereology in Metal, Metallurgiya, Moscow (1977) 376p. (in Russian) [К.С. Чернявский. Стереология в металловедении. М.: Металлургия. 1977. $376 \mathrm{c}]$

7. E. V. Kozlov, N.A. Koneva, et.al. Russ. Phys. J. 45 (3), 285 - 302 (2002) (in Russian) [Э. В. Козлов, Н. А. Конева. Изв. ВУЗов. Физика. 45 (3), 52 - 71 (2002)]

8. E. V. Kozlov, L. I. Trishkina, N.A. Koneva, Crystallogr. Rep. 54 (6), 1033 - 1042 (2009) (in Russian) [Э. В. Козлов, Л.И. Тришкина, Н.А. Конева. Кристаллография. 54 (6), 981 - 990 (2009)]

9. L. I. Mirkin, Physical Basics of Strength and Plasticity MSU, Moscow (1968) 538p. (in Russian) [Л. И. Миркин. Физические основы прочности и пластичности. М.: Изд-во МГУ. 1968. 538 с]

10. N.A. Koneva, L.I. Trishkina, E. V. Kozlov, Izv. Vyssh. Uchebn. Zaved., Fiz., 8, 33-46 (2011) (in Russian) [Н.А. Конева, Л.И. Тришкина, Э.В. Козлов. Изв. ВУЗов. Физика. 8, 33 - 46 (2011)]

11. M. R. Staker, D. L. Holt. Acta Met. 20. 569 - 579 (1972)

12. N. A. Koneva, S. F. Kiseleva, N. A. Popova, Structural Evolution and Internal Stress Fields. Saarbrucken: Lambert, Academic Publishing (2017) 148p. (in Russian) [Нина Конева, Светлана Киселева, Наталья Попова. Эволюция структуры и внутренние поля напряжений. Saarbrucken: Lambert, Academic Publishing. 2017. $148 c]$. 